\title{
Analysis on the Ethical Issues in China's Outdoor Sports
}

\author{
Xian Dongjin \\ School of Physical Education of Changzhou University, Changzhou, Jiangsu,China, 213164 \\ Email: xdj@cczu.edu.cn.
}

Keywords: Outdoor sports; Ethic; Analysis; Moral logic; Ethical Issues

\begin{abstract}
The paper has researched the ethical issues in the development of outdoor sports in China by the method of literature and the empirical analysis method. The results of the study show that our outdoor sports have three aspects of ethical issues, being involved with individual participants, group participants and the organizers. The author has further analyzed the reason of the formation of these ethical issues in our outdoor activities, revealed deep ethical root and moral logic, and thus put forward ethical countermeasures to maintain the healthy and sustainable development of our outdoor sports in order to promote the formation of ethical behaviors for the construction of socialist culture with Chinese characteristics.
\end{abstract}

\section{Introduction}

Around the 1980s, outdoor sport was introduced to China from the developed European countries and the United States. Since then, with the continuous improvement of our national economic level and the acceleration of the process of modernization, outdoor sports entered into a rapid development period. The outdoor sports have entered into our country for only a short time, and organizers and participants have uncertain issues on concept, physical fitness, techniques, management and operation and other aspects, so that there appear some ethical issues that are not conducive to the healthy development of outdoor sports in China. In order to maintain the healthy, harmonious and sustainable development of our outdoor sports, we should strength the ethical and moral constraints and regulate participants' behavior in participating in outdoor activities. It is imperative to carry out the ethical research on the development course of our outdoor sports in order to promote the physical and mental health of the masses, animate mass cultural and sports life and establish a scientific, healthy and civilized social lifestyle[1].

\section{The Research Object and Method}

The Research Object. Taking the ethical issues of organizers and participants in the development of outdoor sports in China as the research object, the paper mainly has studied the issues, including the absence of individual care among teammates, uncommitted free behaviors, the neglect of life safety and environment responsibility, the absence of moral standards in activity organization and conduct, the lack of professional personnel, the buck-passing after casualty accidents.

The Questionnaire Reliability Test. The questionnaires were mainly issued in colleges and universities in Jiangsu. The author has given some analysis and carding on collected materials. This time the author totally issued 1000 copies of questionnaire and collected 921 copies back. Among them, there are 844 valid questionnaires, with an effective rate of $91.36 \%$. In these samples, there are 537 men and 307 women, within an age range from 18 to 60. The author also contacted the Nanjing Outdoor Association in the investigation process and get opinions from relevant experts, which could provide authoritative data for this research[2]. 


\section{The Research Results and Analysis}

The Ethical Issues of Individual Participants. The uncommitted free behaviors and the neglect of life safety and environment responsibility: in December 2012, eighteen students of Fudan University spontaneously organized to adventure to an unopened area of Mount Huangshan. They entered into the scenic spot without buying tickets. Some of them just wore like a traveler, lacking professional equipment. They called the police for help when they were trapped. When the police helped them get down, the police Zhang Ninghai felt off and died. This is a typical outdoor safety accident due to the uncommitted free behaviors of participants[3]. Similar stories happen at all time in China due to the neglect of life safety and environment responsibility. Some outdoor sports participants throw garbage wherever they go. It may destroy the ability of sustainable development of natural environment. Some, due to the lack of experience, light a bonfire in the dangerous area of the forest, which could bring a great hidden danger and threat to the environment and ecological system. Some destroy nature landscapes by trampling on it, directly damaging the survival ability of natural environment, but it happens everywhere in China..

The Ethical Issues of Group Participants. The absence of trust and care among teammates: due to some restrictions on time, place, equipment and etc., most of outdoor activities, except for activities organized by some already-existing mature outdoor sports groups and organizations, are organized and carried out by individual initiators through network, telecommunication and other approaches. Some commercial clubs absorb new members ceaselessly to join their clubs, so as to operate and develop their organization. They gather a lot of unacquainted outdoor sports enthusiasts in order to carry out outdoor activities jointly. However, these participants have no mutual trust and confidence on each other, let alone care about each other[4].

The Ethical Issues of Organizers. The absence of moral standards in activity organization and conduct and the lack of professional personnel: the outdoor sports were introduced to our country in a short time, and new moral standards have not been fully established, so our outdoor sports organizers have no clear moral principles to follow. There is no specific moral principle in the outdoor activities organized by clubs and folk spontaneous organizations. They manage at will and organize activities according to personal preference, use new stunts to attract participants without the slightest consideration of danger, and take money-saving and trouble-saving as principle. Once the team started, they may think the completion of their task. If there is any accident, they would be responsible for nothing[5]. The majority of outdoor activities basically do not get official permission, examination or intervention. In most of the organizations, there is no professional personnel or safety protection personnel to operate and manage outdoor activities. In a disorder and spontaneous form, these organizations have no property safety or responsibility consciousness. They only care about how to carry out activities, how to get business income, how to become famous, without considering the teaching of first aid skills, the selection of first aid equipment, the supply of professional personnel. These are the biggest security risks of outdoor activities.

\section{The Causes of Ethical Issues of Outdoor Sports in China}

Outdoor Sporters Have No Strong Safety Awareness and Pay Less Attention to Professional Skills and Activity Experiences. Less safety awareness has become a common feature of outdoor activity participants, and it also is the main reason of outdoor sports accidents. From the viewpoint of ethics, all moral behavior of people is at the disposal of moral consciousness. Participants often only see the wonderful and exciting, healthy and happy aspects of outdoor activities, thinking of outdoor sports just as simple walking and running and jumping or playing equipment and tools, but they ignore the outdoor security, so they do not produce a sense of crisis, and not have safety awareness yet. In this case, their behavior is conducted without sense of responsibility. Lack of safety responsibility lies on the unreasonable tactics, nonstandard technical application and less experience in specific activities. 
Less professional skill and activity experience resulted from lack of sense of safety responsibility is just like a unarmed person in outdoor activity is exposed to the danger, vulnerable to natural hazards and even fatalities may occur in a relatively safe condition[6].

The Moral Education for Outdoor Sports is Lagged. As a new sports field, outdoor activities passed the moral content in lack of spirit of life and the times, it keeps theory disconnected with practice and simply can not cope with the phenomenon of moral deficiency. While it regards moral education as moral knowledge education, simply imparting the relevant ethical requirements and specifications, but not giving attention to the effect of education, as as to make the moral education conducted only in the formality. As the guide of moral education, the public opinion of outdoor sports should have positive energy, help to the healthy development of outdoor sports, and take moral cultivation of individual as the power to improve and develop outdoor sports, to which we should pay great attentaion. For the shortage of such contents, especially for the lack of public opinion on individual morality of outdoor sports, there is no effect of model, which provids an opportunity for moral abnormality of outdoor sports and even brings a condition for moral corruption[7].

Moral Abnormality of Outdoor Sports. Shortage of professional ethics in rules results in the nonstandard morality management of outdoor sports organizers, to make the organizers speculate in the system implementation and to reduce the professional conduct. Essentially on the professional ethics, sense of professional responsibility is the foundation for the relative personnel of outdoor sports to fulfill various obligations for outdoor sports, individual, society and environment, but the ethics control mode of outdoor sports has not completed in our country, so the sense of professional responsibility can not be defined for the individuals. Outdoor activities are based on entertainment so it is difficult to make the relevant personnel produce professional responsibility.

Lack of Ethics Evaluation System for Outdoor Sports. The so-called responsibility principal in ethic abnormality of outdoor sports is a complete system that contains the participants, organizers, enterprises and the government. As it is unclear that who shall take the major responsibility for the accident in outdoor activities, sometimes task division is not clear, responsibility is absent, command is not obeyed, and action is reckless in the outdoor activities, which is just the portent of an outdoor sports accident. Furthermore, once the outdoor accidents occur, from a viewpoint of economy, it is hardly determined who shall answer for the economic compensation and who shall deal with problems from the accidents. In fact, by the information available, the cost arising from rescue personnel and the like, is born by the public purse basically, which is undoubtedly an important reason of the disorderly development of outdoor sports[8].

\section{Ethical Solution of Healthy Development of China's Outdoor Sports}

To Trust and Obey Team. To trust and obey team in an orderly activity, is one of the important norms of outdoor sports ethics. It requires participants of outdoor sports trust their teammates, obey their team leadership in the practice, orderly conduct the team plans, share the team task and complete their own obligations, to make contributions to the successful completion of project goal of the team. This norm reflects the conformity of trust and obedience with orderliness, represents the lofty struggling and sacrificial spirit of participants for the development of the team, which is significant for the reviviscence and moral progress of participants[9].

To Be Understanding and Tolerant of Teammates. To be understanding and tolerant of teammates in a happy activity, is one of the basic norms of outdoor sports ethics. In the participation of outdoor activities, more or less interaction between individuals will be required, but the happiness from the interaction is one of the goals of individuals to participate in outdoor sports. The activities are carried out in a happy manner only when the individuals are satisfied in psychology and spirit through mutural understanding and tolerance. 
To Enthusiastically Help Teammates. To enthusiastically help teammates in a harmony activity, is one of the basic norms of the most normal outdoor sports ethics. When respect, understanding and care are recognized among the group members, enthusiasticlly helping teammates will become a normal ethical behavior among all the interior members. Helping each other of team members in a wholly-hearted, active and brave state, helps the guaranty of harmonious implementation of team activities. The harmonious implementation of team activities is an important feature of an excellent outdoor sports team.

To Keep Discipline. To keep discipline in a safe activity, is a common guideline for the participants of outdoor sports to be responsible for themselves, others and the organization in the outdoor activities, which reflects their great attention on safety, their love and respect for life. For the safe implementation of outdoor activities, we are required to set up awareness of safety that is the embodiment of keeping discipline. In the outdoor sports, discipline-keeping responsibility is determined in the way of ethical norms, to make the participants enhance their physical and technical training before and during the outdoor activities, build their capacity to initiatively adapt to the outdoor conditions, temper themselve, take exercise, consolidate knowledge and improve quality, so that they have "real capability" to keep discipline all the times, and whether in climbing mountains or wading, they always remind the importance of their safety, and then they will overcome the difficulties and go ahead bravely[10].

To Take away Garbage from Activities. To take away garbage in a green activity, is a ethical norm of special significance to conduct outdoor sports. Taking away garbage is one of the green conduct types of outdoor activities, which also include caring for plants and animals, no bonfires at random and keeping away from the animal habitats. True and perfect outdoor sports must rely on natural conditions, but how to conduct a "green" outdoor activity, first depends on the consciousness and secondly the specific behavior, both indispensable, of which the consciousness dominating the behavior is particularly important.

\section{Conclusion}

In order to form a relative mature, healthy and orderly development and circulation mode of outdoor sports, we need reasonable and strong mechanism support as well as individual as operating cell to restrain self, evaluate self, regulate self and reflect on self, thus forming an ethical mechanism from the inside out. In this way, we can maintain the healthy development of outdoor sports. It has an extremely important meaning to our national sports cause in the first stage and the development of our national cultural construction cause to develop the the ethics of outdoor sports. In order to keep a sound development of outdoor sports, we should insist on the dominant role of the relevant government departments and play the cooperative role of all walks of life. The industrial insiders should adhere to the basic guiding principle of people orientation, overall consideration, risk prevention and accord development. Consequently, we can dissolve all prominent ethical issues in our outdoor sports, build a harmonious environment for outdoor sports and speed up the pace of the construction of socialist culture.

\section{References}

[1] Gong Zhengwei. Building of Contemporary Chinese Sports Ethics [M]. Beijing: Beijing Sports University Press, 2009

[2] Zeng Jianping. Ethical Reflection on Green Sport [J]. Morality and Civilization, 2007 (1): 29-32

[3] Tao Qian, Liang Haifei. Sport Ethics and Harmonious Society [J]. Sports Culture Guide, 2005 (10): $38-40$ 
[4] Ye Changqing. Review on "Green Education" Course Reform of Outdoor Sports of Colleges in View of "Green University" [J]. Zhejiang Sports Science, 201133 (2): 85-87

[5]Li Jianning, Evaluation Research on Discipline Competitiveness of Universities [D], Shanghai, East China Normal University, 2004: 18- 26.

[6]Du Gang, Cui Ting, Study on Levels of the Core Competence of Enterprise - Dimension Structure as well as Its Evaluation and Determination Model [J], Science of Science and Management of S\&T, 2005(1): 138- 142 .

[7]C.K. Prahalad, G. Hamel, The Core Competence of the Corporation [J], Harvard Business Review, 1990(6): 79- 91.

[8]Zhang Shisen, Ouyang Yun, Core Competency of MBA of Harvard [M], Hohhot, Yuanfang Press, 2003: 6- 7, 135.

[9]Zhang Maolin, Yu Dinghai, Core Competence and Cultivation Strategy of Traditional Ethnic Sports Discipline in Institutes of Physical Education [J], Journal of Shanghai University of Sport, 2010, 4(1): $58-61$.

[10]Liu Quan, Zou Xiaodong, Exploration of the Core Competence of University Discipline [J], Liaoning Education Research, 2004(2): 21. 